\title{
Catecholamine levels in plasma and CSF in migraine
}

\author{
F Martínez, J Castillo, J Pardo, M Lema, M Noya
}

\begin{abstract}
There is clinical and pharmacological evidence of the existence of sympathetic dysfunction in migraine. Adrenaline and noradrenaline concentrations were determined in plasma and CSF of patients during attacks of common or classic migraine, comparing them with controls suffering from stress. Plasma noradrenaline levels were significantly lower in the patients with common migraine than in controls $(p<0.05)$. Other catecholamine levels in plasma and CSF in both migraine groups were only slightly lower than in controls. Our results suggest that central sympathetic dysfunction exists in patients with migraine.
\end{abstract}

(F Neurol Neurosurg Psychiatry 1993;56:1119-1121)

The mechanism of pain and vascular changes in migraine is unknown. There is evidence that central monoaminergic systems are altered before and during attacks. Recent hypotheses propose that central catecholaminergic pathways play a role in migraine. Potentiators of the migraine attack may lower the threshold of the orbitofrontalbrainstem projections that influence the intrinsic noradrenergic system. ${ }^{1}$

Electrophysiological, ${ }^{2}$ clinical, ${ }^{3}$ and pharmacological ${ }^{4}$ studies suggest central and peripheral sympathetic hypofunction in migraine. Abnormal reflex responses demonstrating this deficit correlate well with low noradrenaline levels. ${ }^{3}$ Biochemical data have been conflicting, showing high, ${ }^{5}$ normal, ${ }^{3}$ or low $^{6}$ levels of catecholamines in body fluids. Little is known about catecholamine levels in CSF in migraine. We have measured adrenaline and noradrenaline concentrations in patients with common and classic migraine during crises, comparing them with control subjects suffering from physical or psychological stress.

\section{Material and methods \\ SUBJECTS}

Patients were studied in the headache unit of the neurology department of Galicia General Hospital, Santiago de Compostela University, Spain. Informed consent was obtained from subjects after explanation of the procedures. We included 16 patients with common migraine, 11 with classic migraine, and 21 controls. Diagnosis of common or classic migraine was made following criteria of the headache classification committee of the International Headache Society. ${ }^{7}$ The control group was composed of patients suffering physical or psychological stress, with the following diseases: patients immediately before surgical intervention ( $n=9)$, multiple sclerosis $(n=2)$, acute stroke during the 24 first hours $(n=8)$, Guillain-Barré syndrome after the acute phase $(n=1)$, and lymphoma $(n=$ 1). Exclusion criteria for patients and controls were hypertension, psychiatric disease, epilepsy, and intake of antidepressive and adrenergic drugs or calcium channel blockers. Smokers were excluded. Controls with abnormalities in CSF (cell count, and protein and glucose content) were excluded. Mean ages (SD) of common and classic migraine patients and controls were $38.37(11 \cdot 7), 39$ $(11.03)$ and $49.95(11.40)$ years. The male/female ratios of the three groups were $0.33,0.57$, and 1.62 . Duration of migraine history was $19.43(10.26)$ years in common migraine and $12.72(5.46)$ in classic migraine. Severity of headache was evaluated by the score units measure (SUM), expressed in headache units, according to international criteria. ${ }^{8}$ Duration of crisis was recorded as the time from onset of headache until sample extraction.

\section{ANALYTICAL METHOD}

Blood collected by venepuncture after 30 minutes' rest in supine position was centrifuged ( $3000 \mathrm{~g}$ for 15 minutes). Lumbar puncture was performed with patients in left lateral decubitus after 30 minutes' rest. Initial pressure was measured and $5 \mathrm{ml}$ of CSF were discarded. The sixth $\mathrm{ml}$ was used for cell count and protein and glucose analysis. The next $5 \mathrm{ml}$ of CSF were centrifuged $(2000 \mathrm{~g}$ for 10 minutes) and used for catecholamine assay. Plasma and CSF samples were stored $\left(-70^{\circ} \mathrm{C}\right)$. Extractions were performed between 9 and 10 am in controls after an 8 hours fast. In patients with migraine extractions were carried out at variable hours, during an attack and after a minimum 4 hours fast. Measurement of catecholamines was performed using high pressure liquid chromatography with electrochemical detection 
(HPLC-EC) and extraction by activated alumina (Bioanalytical Systems Inc, LCEC Appl Note 14). A plasma sample of $1.5 \mathrm{ml}$ was placed in a glass tube with $25 \mu$ l of DHBA $(100 \mathrm{ng} / \mathrm{ml})$ as internal standard and $50 \mathrm{mg}$ of alumina. DHBA was prepared by dissolving $16 \mathrm{mg}$ of DHBA-HBr in $100 \mathrm{ml}$ of $0.1 \mathrm{M}$ $\mathrm{HClO}_{4} ; 100 \mu \mathrm{l}$ of this solution were diluted to $100 \mathrm{ml}$ with $0.1 \mathrm{M} \mathrm{HCIO}_{4}$. Buffer Tris $(1.5$ $\mathrm{M}, \mathrm{pH} 8.6$ ) was added and the mixture agitated, then centrifuged, and the supernatant removed. The alumina was washed three times and removed via filtration. Catechol species were desorbed from the alumina adding acidic buffer $\left(\mathrm{CIO}_{4} \mathrm{H} 0 \cdot 1 \mathrm{M}\right)$. After agitation and centrifugation, $50 \mu \mathrm{l}$ of the acidic supernatant were injected into the HPLC-EC system. Chromatography equipment characteristics: Pump Gilson 305, column Spheriscorb ODS-1, amperometric detector $\mathrm{LC}-4 \mathrm{~B}$ (+600 $\mathrm{mV}$ versus $\mathrm{Ag} / \mathrm{AgCl}$ ), flow rate of $0.8 \mathrm{ml} / \mathrm{min}$. The mobile phase was prepared with monochloride acetic acid, 1-octane sulphonic acid, OHNa and $\mathrm{Na}_{2}$ EDTA. Results are expressed in $\mathrm{pg} / \mathrm{ml}$.

STATISTICAL ANALYSIS

Intergroup differences in plasma noradrenaline were studied using Kruskal-Wallis and

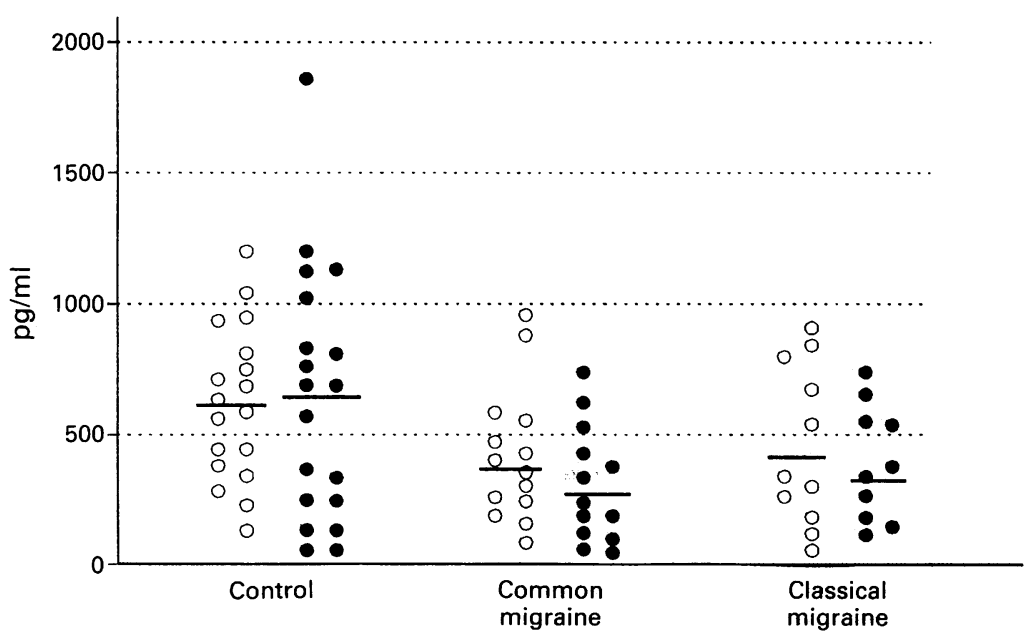

Figure 1 Adrenaline $(\bigcirc)$ and noradrenaline $(\circlearrowleft)$ concentrations in plasma ( $\mathrm{pg} / \mathrm{ml})$. Mean level (-).

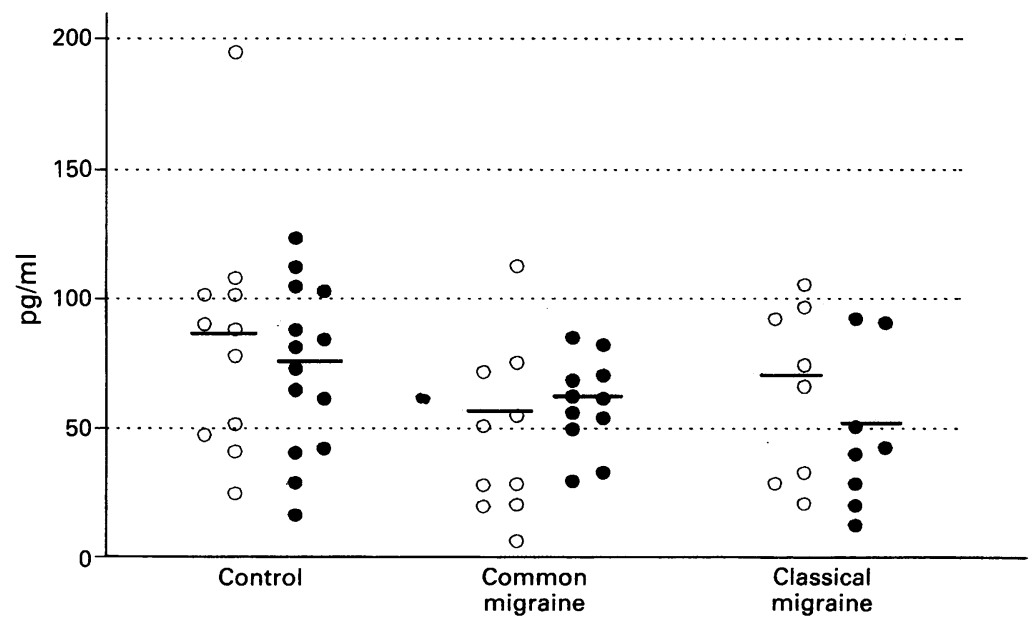

Figure 2 Adrenaline $(O)$ and noradrenaline $(\Theta)$ concentrations in CSF ( $\mathrm{pg} / \mathrm{ml})$. Mean level (-).
Mann-Whitney tests. Comparison of CSF noradrenaline, and plasma and CSF adrenaline was performed with one way analysis of variance (ANOVA) and Duncan's multiple range test. Influence of age and sex was investigated by linear regression and one way ANOVA. The relation between catecholamine levels and severity of headache (SUM) or duration of crisis was investigated with linear regression (power of regression evaluated by the determination coefficient ( $r$ square) and ANOVA).

\section{Results}

Mean (SD) plasma adrenaline levels were $434.9(238.9), 453.9(259.3)$, and 619.6 $(273.2) \mathrm{pg} / \mathrm{ml}$ for common and classic migraine and controls. CSF adrenaline levels were $53.3(32 \cdot 3), 65.4(29.8)$ and $86.2(47 \cdot 0)$ in patients with common and classic migraine and controls. Noradrenaline plasma levels were $308.6(208 \cdot 2), 378 \cdot 3(224 \cdot 3)$, and $651 \cdot 7$ (482.6). Mean CSF noradrenaline concentrations were $59 \cdot 1(15.8), 50.7(27 \cdot 2)$, and $73 \cdot 1$ $(32 \cdot 1)$ for common migraine, classic migraine, and control patients, respectively. Scattergrams with distribution of results in plasma and CSF are shown in figures 1 and 2. We did not observe a significant difference in plasma (figure 1) or CSF (figure 2) adrenaline levels between patients with common and classic migraine, or between migraine groups and controls. Plasma noradrenaline was significantly lower in common migraine patients than in controls $(p<0.05)$ (figure 1$)$. We did not observe differences in plasma noradrenaline levels between both migraine groups or between classic migraine and controls. No significant differences in CSF noradrenaline levels were found between groups (figure 2). No influence of age or sex over catecholamine levels was observed. We found no correlation between noradrenaline levels in plasma or CSF and severity of headache (SUM): $r=0.42, r=-0.03$ (plasma, common, and classic migraine); $r=0.26, r=0.25$ (CSF, common and classic migraine). We also did not find correlation between plasma adrenaline levels (in both migraine groups) or CSF adrenaline levels in common migraine and severity of headache. We found a significant positive correlation between adrenaline levels in CSF and severity of headache in the classic migraine group: $r=0.89, \quad r^{2}=79.22 \%$, $\mathrm{p}<0.01$.

\section{Discussion}

Monoamine levels in $\mathrm{CSF}^{9}$ and plasma ${ }^{10}$ may reflect monoaminergic activity. We have measured catecholamine levels in plasma and CSF of patients with common and classic migraine during attacks using HPLC-EC. Variability of interlaboratory results depend on the sensibility of the method, and the interference of acid or oxidating substances. ${ }^{11}$ This problem has occurred with a proportion of samples in our study.

Important variability of results was 
observed. Levels in CSF were lower than in plasma for both catecholamines in the three groups. We have standardised as many external potential factors that influence sympathetic function as possible. Plasma and CSF levels have been lower than those previously reported in resting controls. ${ }^{12}$ Mean catecholamine levels in plasma or CSF were lower in migraine groups than in controls. Differences were not statistically significant except when comparing noradrenaline levels in plasma between common migraine patients and controls $(p<0.05)$. Our results may suggest that during migraine attacks catecholamines are defectively released, although further studies are required. Though precise location of the defect cannot be determined, results in plasma and CSF suggest both a central and peripheral deficit. We observed correlation between adrenaline levels in CSF and severity of headache in patients with classic migraine.

Previous reports of catecholamine levels in migraine have been conflicting. ${ }^{356} \mathrm{CSF}$ studies have focused upon catecholamine metabolites. Lower levels than in healthy controls have been reported, during and between crisis. ${ }^{35}$ Biochemical data correlate with clinical studies that demonstrate sympathetic dysfunction. ${ }^{3}$ The mechanism by which this dysfunction may participate in the physiopathology of migraine remains elusive. Indirect evidence shows that the noradrenergic system represents the threshold for the attacks. Potentiators of migraine probably modulate sympathetic activity. ${ }^{13}$ Clinical and biochemical results suggest that migraine patients have an abnormal adaptative reaction to stress. ${ }^{14}$ Central noradrenergic systems modulate nociceptive transmission to the central nervous system. Their dysfunction may cause perturbation of these pain modulating systems. ${ }^{15}$ Also, these cerebral pathways participate in the regulation of cortical blood flow. Chronic sympathetic hypofunction may lead to denervation supersensitivity of cerebral vessels. Triggering factors may then cause overconstriction, promoting the vascular phenomena observed in migraine. ${ }^{3}$

1 Welch KMA. Migraine. A biobehavioral disorder. Arch Neurol 1987;44:323-7.

2 Nagel-Leiby S, Welch KMA, Grunfeld S, D'Andrea G. Central catecholamine-dependent neurophysiology in common versus classic migraine. Ann Neurol 1984; 24:161.

3 Havanka-Kanniainen H, Juujärvi K, Tolonen U, Myllylä VV. Cardiovascular reflexes and plasma noradrenaline levels in migraine patients before and during nimodipine levels in migraine patients before and
medication. Headache 1987;27:39-44.

4 Munari L, Milanesi I, Silvani A, Bussone G, Boiardi A. Pharmacologic evaluation of cardiovascular reflex responses in migraine patients: lack of central sympathetic modulation? Funct Neurol 1989;4:375-8.

5 Anthony $M$. Biochemical indices of sympathetic activity in migraine. Cephalalgia 1981;1:83-9.

6 Takeshima T, Takao Y, Urakami K, Nishikawa S, Takahashi K. Muscle contraction headache and during the cold pressor test. Cephalalgia 1989;9:7-13.

7 Headache Classification Committee of the International Headache Society. Classification and diagnostic criteria Headache Society. Classification and diagnostic criteria
for headache disorders, cranial neuralgias and facial pain. Cephalalgia 1988;8(Suppl 7):1-96.

8 Diamond S, Medina JL. Double blind study of propranolol for migraine prophylaxis. Headache 1976;16:24-7.

9 Scheinin M. Monoamine metabolites in human cerebrospinal fluid: Indicators of neuronal activity? Med Biol 1985;63:1-17

10 Goldstein DS, McCarty R, Polinsky RJ, Kopin IJ Relationship between plasma norepinephrine and sympathetic neural activity. Hypertension 1983;5:552-9.

11 Wester P, Bergstrom U, Eriksson A, Gezelius C, Hardy J, Winblad B. Ventricular cerebrospinal fluid nomoamine transmitter and metabolite concentrations reflect human brain neurochemistry in autopsy cases. $f$ Neurochem 1990;54:1148-56.

12 Davis BA. Biogenic amines and their metabolites in body fluids of normal, psychiatric and neurological subjects. fluids of normal, psychiatric and

13 Welch KMA, Levine SR, D'Andrea G. The pathogenesis of migraine. Current Opinion in Neurology and Neurosurgery 1988;1:183-8.

14 Nappi G, Martignoni E, Micieli G, et al. Sympathergic/ neuroendocrine reactivity as a marker of adaptative processes underlying migraine susceptibility. Cephalagia 1987;7(Suppl 6):533-5.

15 Besson JM. Anatomical and biochemical strategy of pain suppressing systems. Cephalalgia 1987;7(Suppl 6): 